\title{
Transition in incompressible boundary layers with two-dimensional excrescences
}

\author{
B. J. McKeon, A. M. Bender† R. V. Westphal ${ }^{\ddagger} \&$ A. Drake ${ }^{\S}$
}

\begin{abstract}
An experimental investigation of the transition process in boundary layers subjected to forward- or aft-facing two-dimensional step excrescences is described. The objective of the work was to characterize the variation of transition Reynolds numbers with measurable roughness and boundary layer parameters, with the specific goal of specifying new tolerance criteria for laminar flow airfoils, alongside a fundamental investigation of linear boundary layer stability mechanisms. Results from an ongoing program of increasing complexity on effects of pressure gradient on excrescence-induced transition are presented. Preliminary $\mathrm{N}$-factor calculations are used to determine the effects of boundary layer stability and attempt to isolate the effect of the disturbance due to the excrescence.
\end{abstract}

\section{Nomenclature}

$k \quad$ step excrescence height

$s \quad$ surface distance from leading edge

$x \quad$ streamwise distance

$A(x)$ instability amplitude at streamwise location $x$

$A_{0} \quad$ initial instability amplitude

$C_{f} \quad$ skin friction coefficient

$K \quad$ acceleration parameter, $\frac{\nu_{e}}{U_{e}^{2}} \frac{d U_{e}}{d x}$

$N \quad$ exponential amplification factor

Re Reynolds number based on freestream velocity, $1 / \nu \int U_{e}(s) d s$

$U_{\infty}$ freestream velocity

$\alpha \quad$ disturbance wavelength

$\gamma \quad$ disturbance amplification rate

$\delta^{*} \quad$ displacement thickness

$\nu \quad$ kinematic viscosity

$\omega \quad$ disturbance frequency

$\theta \quad$ momentum thickness

Subscript

crit for transition to occur

$e \quad$ local freestream conditions

$k \quad$ at excrescence location

$t \quad$ at transition

$x \quad$ at local streamwise location

$\infty \quad$ upstream freestream conditions

*Assistant Professor of Aeronautics, Graduate Aeronautical Laboratories, California Institute of Technology, Pasadena, CA 91125. Member, AIAA.

${ }^{\dagger}$ Northrop Grumman Corporation, El Segundo, CA 90245

${ }^{\ddagger}$ Professor, School of Mechanical \& Materials Engineering, Washington State University, 2710 University Drive, Richland, WA 99354. Senior Member, AIAA.

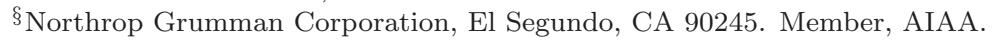




\section{Introduction}

\section{I.A. Background to the work}

Transition of the boundary layer from a laminar state to a turbulent one is accompanied by a dramatic increase in drag, thus designers of low-drag aero-vehicles seek to maximize the extent of the laminar part of the boundary layer. This is a topic of continuing importance with the extensive current interest in Unmanned Aerial Vehicles (UAVs). Many long-endurance aircraft are designed to exhibit large extents of laminar flow relative to the small chord of the airfoils. In flight, however, conditions may be quite removed from ideal, due to either environmental conditions or deviation of the airfoil surface from a smooth finish. Considerable importance is attached to determining the influence of surface protuberances on the transition process in order to determine criteria for manufacturing tolerances, since the complete elimination of all roughness elements is prohibited by manufacturing complexity and cost.

Roughness on real airfoils can consist of isolated three-dimensional elements, distributed roughness or twodimensional excrescences, arising, for example, due to damage, in-flight deposits or finite, fixed manufacturing tolerances on the surface. The latter phenomenon, perhaps a mismatch between adjacent wing panels, can be well-modeled by two-dimensional, step-like excrescences on a flat plate. A favorable external pressure gradient is more realistic to conditions close to the leading edge of real airfoils and will likely lead to less stringent requirements on the step sizes by simple stability arguments. Identifying and understanding these permissible step sizes is of considerable importance for the efficient manufacturing and operation of laminar flow vehicles. The academic problem relates to describing the change in the transition characteristics of the boundary layer induced by two-dimensional roughness elements of varying geometry in the presence of pressure gradients.

For the quiet environments of flight (low turbulence level with broadband disturbance spectrum), prediction of transition is commonly performed using the $e^{N}$ transition prediction method ${ }^{1,2}$, which assumes that transition occurs when the overall amplification of Tollmien-Schlicting waves reaches a particular value of $N \approx 7-10$ from the literature, where

$$
N=\ln \left(\frac{\mathrm{A}}{\mathrm{A}_{0}}\right)=\max _{\omega} \max _{\alpha} \int_{\mathrm{x}_{\mathrm{i}}}^{\mathrm{x}_{\mathrm{t}}} \gamma(\mathrm{s} ; \omega, \alpha) \mathrm{ds} .
$$

Here $x_{i}$ is the streamwise location corresponding to the critical Reynolds number for boundary layer instability, $x_{t}$ is the location of transition and $\gamma(x ; \omega, \alpha)$ is the peak disturbance amplification rate, a function of disturbance frequency $\omega$ and wavelength $\alpha$. The receptivity of the boundary layer to external disturbances sets the initial amplitude, $A_{0}$, with details of the absolute initial amplitude calibrated into the $N$ factor that is required for transition in a given disturbance environment. ${ }^{3}$

The influence of small surface excrescences on the transition process has been considered as a local stability modification as a result of change in boundary condition ${ }^{4}$ or the perturbation pressure field ${ }^{5,6}$, with a downstream return to amplification curves that parallel the undisturbed rates (if transition does not occur before this relaxation can occur). In summary, the local amplification rate increases. Of course, if the roughness is small enough it may have negligible effect on the transition location. For larger amplitude disturbances, separation may occur, ultimately leading to transition in the separated shear layer. Excrescences may also act as receptivity sites, leading to increased disturbance amplitudes at the excrescence ${ }^{7,8}$. So long as transition does not occur too close to the excrescence, the effect has been described by a roughnessdependent change to $N, \Delta N$ (as detailed by, e.g. ${ }^{8,9}$ ) with considerable success, more or less independent of the specifics of the boundary layer under test.

\section{I.B. Dimensional considerations}

For a given disturbance field and a zero pressure gradient, dimensional considerations yield

$$
\delta_{t}^{*}=f\left(k, \delta_{k}^{*}, U_{\infty}, \nu\right)
$$

where $\delta_{t}^{*}$ and $\delta_{k}^{*}$ are the displacement thicknesses of the boundary layer at transition and at the excrescence location, respectively, $k$ is the excrescence height, $U_{\infty}$ is the local freestream velocity and $\nu$ is the kinematic viscosity. Then, for example,

$$
R e_{\delta^{*}, t}=f^{\prime}\left(\frac{\delta_{k}^{*}}{k}, R e_{\delta^{*}, k}\left(\text { or } R e_{k}\right)\right)
$$

$$
2 \text { of } 10
$$


where $R e_{\delta^{*}, t}=\frac{\delta_{t}^{*} U_{\infty}}{\nu}, R e_{k}=\frac{k U_{\infty}}{\nu}$ and $R e_{\delta^{*}, k}=\frac{\delta_{k}^{*} U_{\infty}}{\nu}$.

A combination of non-dimensional variables that renders the effect of one to be small is sought, with a traditional goal being the determination of a critical roughness Reynolds number, $R e_{k, c r i t}$, below which the excrescence does not influence the transition location. $R e_{k, c r i t} \approx 900$ has been suggested by Dryden ${ }^{10}$ and $\mathrm{Tani}^{7}$ for cylindrical, two-dimensional roughness elements, based on a review of all incompressible boundary layer data available at the time. Perraud \& Seraudie ${ }^{11}$ demonstrated $R e_{k, c r i t} \approx 900$ for aft-facing steps and $R e_{k, c r i t} \approx 1800$ for forward-facing ones. Wang \& Gaster ${ }^{9}$ and Crouch et al ${ }^{8}$ have had recent success considering the variation of $R e_{x, t}$ with $k / \delta_{k}^{*}$ over relatively small ranges of $R e_{\delta^{*}, k}$.

In the presence of an external pressure gradient, the boundary layer stability characteristics are modified from the Blasius case. Favorable gradients lead to stabilization, increasing the critical Reynolds number for neutral stability $R e_{\delta^{*}, \text { crit }}$ and decreasing peak amplification rates, with the reverse true for adverse gradients, in which the inviscid, inflectional instability can become important. $N$ factor growth in a boundary layer under a pressure distribution that resembles those existing on laminar flow airfoils can be approximated by recourse to the single-parameter Falkner-Skan family of velocity profiles ${ }^{12}$, under the quasi-parallel assumption. The effect of pressure gradients on transition in the presence of blended step excrescences will be described here.

\section{I.C. Previous work}

The literature on this topic is broad and a brief review of mostly recent work is given below.

The experimental work of Wang \& Gaster ${ }^{9}$ has addressed the effect of forward- and aft-facing step changes in surface height on the transition location in zero-pressure gradient (Blasius) boundary layers. By changing the unit Reynolds number via the freestream velocity and the roughness height while maintaining a constant streamwise step location they achieved parameter ranges of $x_{k} / k \leq 150$ and $1000<R e_{\delta *, k}<1400$, approximately, with $R e_{x, t} \leq 2.4 \times 10^{6}$. The transition Reynolds number was determined using the streamwise location at which spikes indicative of nonlinear breakdown were first observed in the signal from a hot-wire placed at the outer edge of the boundary layer. The data demonstrated good collapse to a single curve of $R e_{x, t}$ vs $k / \delta *_{k}$ for each type of step and, consequently, single curves of the change in $N$-factor, $\Delta N$, associated with forward- and aft-facing steps. Backward-facing steps led to larger reductions in transition Reynolds number for a given non-dimensional step height, consistent with the impact of flow separation.

Crouch, Kosorygin \& $\mathrm{Ng}^{8}$ performed an experimental study of the influence of forward- and aft-facing steps at two streamwise locations corresponding to a favorable and adverse pressure gradient in a prescribed pressure distribution, using the minimum in measured Preston probe pressure to specify the location of transition. No consistent trends in the variation of transition Reynolds numbers between the different types of pressure gradient can be determined in their plots of $R e_{x, t}$ vs $k / \delta_{k}^{*}$, but the two general curves for the $\mathrm{N}$-factor corrections introduced by forward- and aft-facing steps as a function of $k / \delta_{k}^{*}$ indicate that the favorable pressure gradient leads to more scatter in $\Delta N$, bounded by the same approximate curve as for the adverse pressure gradient.

The computational work of Nayfeh ${ }^{6}$ determined the pressure distribution associated with steps and humps and the corresponding stability analysis indicated regions of strong stabilization associated with even short local acceleration regions caused by the excrescences. Perraud \& Seraudie ${ }^{11}$ made experimental and computational determinations of the critical Reynolds numbers and changes to $\Delta N$ for Blasius and airfoil boundary layers.

Tani ${ }^{13}$ summarized work on two-dimensional cylindrical roughness elements, again obtaining single curves for a given disturbance environment. He noted that the influence of background turbulence level on the $R e_{x, t}$ vs $k / \delta_{k}^{*}$ curves was confined to the low non-dimensional step heights, corresponding to a lower undisturbed transition Reynolds number.

The influence of the different transition indicators, namely the onset of turbulent spikes ${ }^{9}$ versus pressure measurements such as Preston probe indicators of a minimum in dynamic pressure followed by a rise in wall shear stress $,{ }^{8},{ }^{13}$ on the empirical scaling results has not been explicitly detailed, but the former will give a more conservative measure of the onset of instability.

In this work, an early part of a larger program on the prediction of manufacturing tolerances for laminar flow (MEATLOAF) ${ }^{14}$ performed by Northrop Grumman and funded by the Air Force Research Laboratory, we investigate the scaling of the transition location in an experimental, incompressible boundary layer subjected to disturbance by two-dimensional blended step surface excrescences at varying Reynolds numbers 
based on displacement thickness and pressure gradients of varying strength. The extended empirical relationships between transition location and excrescence parameters are of interest rather than simply a critical Reynolds number, because of the intended application to laminar flow airfoils.

\section{Approach}

The experiments were performed in the Washington State University Low Speed Wind Tunnel (figure 1) at the maximum freestream velocity achievable in this facility, namely $30 \mathrm{ft} / \mathrm{s}(9 \mathrm{~m} / \mathrm{s})$. Laminar flow could be maintained up to $R e_{x}=1.3 \times 10^{6}$ for a zero pressure gradient boundary layer under the same operating conditions (using a longer plate apparatus). Pressure gradients were imposed by an adjustable height ceiling and measured using a streamwise row of 40 static pressure tappings, $2.5^{\prime \prime}$ from the centerline on the test plate and a rear blockage strip was used to ensure that the stagnation point was slightly on the measurement side of the nose of the leading edge.

The modular plate design (figure 2) had a super-elliptical leading edge and a total length of $4 \mathrm{ft}(1.2$ $\mathrm{m})$, dictated by the test section length. Excrescence inserts with a step change in height of $0.035-0.100$ " at one end and a linear recovery over the full $3^{\prime \prime}$ insert length were used in both forward- and aft-facing configurations at each of the four downstream locations. Henceforth the excrescence location, $x_{k}$, will refer to the streamwise position of the step height change. Thus $x_{k}$ could be varied from $5-14^{\prime \prime}$ for the forwardfacing excrescence and $7-16^{\prime \prime}$ for the aft-facing excrescence. The tolerance on all other insert joins implied negligible additional excrescence heights. The smooth wall boundary layer was laminar the entire length of the plate for all but the adverse pressure gradient cases.

The pressure gradients were designed to yield approximately constant values of the acceleration parameter, $K=\nu_{e} / U_{e}^{2} d U_{e} / d x$, over the bulk of the plate, with the exception of the leading edge. The distribution of pressure coefficients and velocities for the cases detailed here are described in figure 3, and the acceleration parameters are shown in figure 4. This represents a simpler practical pressure gradient scaling than determining the equivalent local Falkner-Skan parameter (e.g. Gaster \& Jiang ${ }^{12}$ ).

All pressure measurements were made using an MKS Baratron model 698 powered by a model 270D signal conditioner. The transducer accuracy was $0.15 \%$ and the zero drift was negated for each set of measurements. The wall-normal position for the boundary layer profiles was set using a Velmex A15 Unislide with $20 \mu \mathrm{m} / \mathrm{step}$ resolution.

Experimental boundary layer profiles were acquired for the smooth plate at each pressure gradient using a total pressure (Pitot) tube with 0.050 or $0.032^{\prime \prime}$ outer diameter at a range of downstream locations covering the various locations of the excrescences and the local surface pressure measurement. Estimates of the local skin friction at each station were determined using pressure measurements from Preston probes of $0.042^{\prime \prime}$ outer diameter under laminar and turbulent calibrations and compared to computed reference distributions. The transition region typically extended over a distance $R e_{x, \text { onset }}-R e_{x, \text { completion }} \approx 10^{5}(x \approx 6$ ") and the transition location was determined to correspond to the position at which the spatially-extrapolated skin friction exceeded the laminar reference at that location by $20 \%$. An example of the determination of the transition location is given in figure 5 .

The experimental set-up did not permit capture of smooth wall on-plate transition for anything other than an adverse pressure gradient (due to the combination of unit Reynolds number and test section length).

Computational studies to examine the detailed flow behavior in the presence of the surface steps were performed using the Northrop Grumman Generalized Compressible Navier-Stokes (GCNS) code, which is widely used for design and the investigation of detailed flow features, for the exact smooth-wall test geometry. Full details of the computations are given in Drake et al, 2005. ${ }^{14}$ The two-dimensional calculations of the laminar boundary layer under the experimental pressure distributions were used here to determine the variation of boundary layer parameters such as the streamwise variation of displacement thickness, $\delta^{*}$, momentum thickness, $\theta$, and skin friction. There was good agreement between the computations and the smooth-wall experimental profiles. Because of the blended nature of the excrescence disturbances, these computations are not exact with respect to the incoming boundary layer for aft-facing steps, but give a useful guide to the condition of the base flow.

The laminar boundary layer computations were used as input to the Rapid $N$-factor analysis described in Gaster et $a l^{12,15}$ in order to perform a preliminary investigation of the stability of the boundary layer under each pressure gradient. This analysis uses Padé approximants and a parallel flow assumption to obtain a functional form for the the neutral stability loop and thus permits rapid, accurate estimation 
of the eigenvalues to determine instability amplification rates across a range of frequencies. Errors are introduced because of the assumptions associated with fitting local Falkner-Skan profiles to the actual flow and the neglect of the streamwise gradient terms, but indicative results can be obtained with accuracy that is expected to exceed that innate to the experimental measurements.

Figure [6 shows the results for a Blasius boundary layer with a constant (representative) freestream velocity of $9.8 \mathrm{~m} / \mathrm{s}$. Clearly transition for the smooth-wall Blasius boundary layer would occur at a downstream distance that exceeds the length of the test article.

\section{Results and Discussion}

\section{III.A. Mildly favorable pressure gradient, P0 $\left(K=0.5 \times 10^{-7}\right)$}

The zero pressure gradient represents the baseline for these experiments, and one for which there exist several data sets for comparison. Under approximately constant freestream pressure conditions a very mildly favorable pressure gradient boundary layer $\left(K \approx 0.5 \times 10^{-7}\right)$ developed, with the relatively low freestream velocity leading to low Reynolds numbers at the excrescence location in the approximate range $450<$ $R e_{\delta^{*}, k}<850$ (where the lower limit is close to the critical Reynolds number for a Blasius boundary layer of $R e_{\delta^{*}, k}=520$ under the parallel flow assumption). The approximate $\mathrm{N}$-factor variation for this flow arising from the disturbance growth rates obtained from Rapid- $N$ stability calculations (shown in figure 7 ) highlight the effects of the leading edge acceleration and deceleration $(x \leq 3$ ") and the slight acceleration downstream of the excrescence site, which act to destabilize and stabilize the boundary layer, respectively, with respect to a true Blasius flow (figure 6 ). Interestingly, the estimated smooth-wall N-factor at the trailing edge was similar in both cases. P0 conditions are, however, close to a zero pressure gradient case and will be used to compare the results to previous work.

Figures 9 and 10 show the variation of $R e_{x, t}$ with non-dimensional excrescence height, $k / \delta_{k}^{*}$, for forwardand aft-facing excrescences at the different streamwise excrescence locations (i.e., different values of $R e_{\delta^{*}, k}$ ). Since transition did not occur on the plate in the smooth wall case, the transition location for $k / \delta_{k}^{*} \rightarrow 0$ has been fixed at the trailing edge, $R e_{x, t}=7.4 \times 10^{5}$, although this is obviously an underestimate of the true value. The results are consistent with the generation of linear instability waves.

The aft-facing step can be seen to cause a stronger, more rapid, reduction in transition Reynolds number than a forward-facing step of the same non-dimensional magnitude, in agreement with earlier observations and indicating that for these values of $k / \delta^{*}$ flow separation occurs at the step. However the transition Reynolds number at the largest value of $k / \delta_{k}^{*}=2$ is approximately the same in both cases as transition reaches the streamwise step location.

There is a clear effect of streamwise location of an excrescence with a given step height, $x_{k} / k$, as $k / \delta_{k}^{*}$ increases because the transition location moves upstream towards the excrescence location, $x_{t} / x_{k}<1.3$, approximately, with transition ultimately likely occurring without generation of T-S waves. Data for each $R e_{\delta^{*}, k}$ deviate from a single curve in agreement with the results of Tani ${ }^{13}$ for constant $x_{k} / k$, in which a minimum transition Reynolds number followed by an approach to the asymptote $R e_{x, t}=R e_{x, k}$ is obtained as $\delta_{k}^{*}$ is decreased via the freestream velocity.

A comparison of the present data with the results of Wang \& Gaster ${ }^{9}$ at zero pressure gradient and Tani $^{13}$ is given in figures 11,12 . The present data more closely resemble the cylindrical wire results of Tani than the pure step of Wang \& Gaster, perhaps confirming the latter's observation that the geometry of the two-dimensional disturbance seems to have a dominant effect on the transition location as a function of roughness height. This is intuitively correct given the region of blending from the mean line to the maximum height on each excrescence insert, and the associated modifications to the boundary layer stability arising from local regions of favorable (adverse) pressure gradients upstream of the excrescence when used as an aft(forward-) facing disturbance, with corresponding changes in the stability of the velocity profile. However, as the $\mathrm{N}$-factor plots show, the P0 boundary layer also has downstream stability characteristics that likely deter transition in the aft section of the plate (this is borne out in the observed transition locations being mostly upstream of the region of rapidly decreasing $\mathrm{N}$-factor), leading to abrupt changes in the transition location. The reality is likely a combination of the two effects.

The present results were also obtained at much lower $R e_{\delta^{*}, k}$ than in previous experiments ${ }^{9}$, meaning that the disturbance is effectively introduced much closer to the critical Reynolds number for instability and closer to the peak amplification rate determined by non-parallel stability calculations ${ }^{16}$, experiments ${ }^{17}$ and Rapid- $N$ analysis. 
In the absence of a determination of the true smooth-wall critical N-factor, $N_{\text {crit }}$, we examine the equivalent smooth-wall N-factor for each transition occurrence, $N_{e q}$ (hence the true change in N-factor associated with a particular excrescence in this flow, at this turbulence level, will be $\Delta N=N_{\text {crit }}-N_{e q}$ ). The preliminary calculations of the variation of $N_{e q}$ with non-dimensional step height is given in figures 13 and 14 for forward- and aft-facing steps, respectively. In both cases the variation of $N_{e q}$ is not monotonic, due to the nature of the smooth-wall N-factor curve. For the forward-facing step, the plot mirrors the trends of a section of the $\mathrm{N}$-factor curve, with reasonable agreement between the data at different values of $R e_{\delta}^{*}, k$. When the step faces aft, transition further upstream than for the forward-facing case skews the curve to N-factors of approximately 4. Note that the influence of $x_{k} / k$ (per figures 9 and [10) will be important for at least the largest step height at each $R e_{\delta^{*}, k}$.

These results suggest that in this flow the effect of a relatively large excrescence on the boundary layer is a sufficiently large local modification to flow stability for large disturbance amplification and transition upstream of boundary layer mean velocity relaxation rather than a step increase in N-factor followed by a return to undisturbed rate of change of amplification factor as observed by Perraud \& Séraudie. ${ }^{11}$ Nayfeh $^{6}$ has shown that even small excrescences can create a small streamwise extent of stability followed by a streamwise region of high amplification rates over an extended frequency ranges in an otherwise unstable Blasius boundary layer.

\section{III.B. Stronger favorable pressure gradient, P8 $\left(K=9.4 \times 10^{-7}\right)$}

Consider now the stronger favorable pressure gradient case, P8, in which the lowest value of the Reynolds number at the step, $R e_{\delta^{*}, k}$, is lower than the P0 case because of the slower boundary layer growth. Together with the enhanced stability of the boundary layer, this meant that the roughness was introduced below the critical Reynolds number. Rapid- $N$ calculations (not shown) indicated that the smooth wall flow was stable over the entire plate. Thus the data at this pressure gradient incorporate the influences of introducing roughness disturbances into a linearly stable flow (i.e., a strong influence of $R e_{\delta^{*}, k}$ and boundary layer history) and enhanced boundary layer stability as well as the effect of the pressure gradient on the flow at the excrescence itself.

The effects of introducing the excrescence at different values of $R e_{\delta^{*}, k}$ (varying the downstream location) can be seen for the P8 case in figure 15 and more clearly for the aft-facing step in figure 16, where transition first occurs on the test article at the largest $k / \delta_{k}^{*}$ for the most upstream excrescence location(recall that the true $k / \delta_{k}^{*} \rightarrow 0$ values of $R e_{x, t r}$ are not captured, so a value of $7.4 \times 10^{5}$ is indicative of transition moving off the back of the plate for smaller excrescence heights).

Once again the influence of an aft-facing step results in a lower transition Reynolds number for a given $k / \delta_{k}^{*}$ than for a forward-facing one, and a stronger $x_{k} / k$ effect is observed (reflecting transition moving into the separated shear layer over the step).

Since the undisturbed flow is stable over the length of the plate, N-factor plots do not reveal any new information for these flow conditions.

\section{III.C. Adverse pressure gradient, P2 $\left(K=-5.2 \times 10^{-7}\right)$}

For the adverse pressure gradient case P2, transition on the smooth wall was indicated at a downstream distance of 11 " from the leading edge, corresponding to $N \approx 6$ from the Rapid- $N$ calculations (figure 8). Excrescences could only be introduced in the two upstream locations to prevent immediate transition at the step. The severity of the pressure gradient resulted in rapid forward movement of the transition point with non-zero step height, reaching the step location for both forward- and aft-facing steps unless the disturbance was introduced at the lowest $R e_{\delta}, k$ location.

\section{III.D. Comparison of pressure gradient results}

Figures 17 and 18 show the variation of the transition Reynolds number with non-dimensional excrescence height for all the pressure gradients cases (P0, P8 and P2 in figure 4) for forward- and aft-facing excrescences. Here the displacement thicknesses are based on the measured boundary layer profiles.

Contrary to the conclusions of Crouch et al, a large pressure gradient effect on the shape of the curve describing transition location as a function of non-dimensional step height is observed, likely due to the larger gradients applied in the present study and the effect of $R e_{\delta^{*}, k}$. Within the scatter that arises due 
to transition approaching the excrescence location and the lack of true transition locations for $k / \delta_{k}^{*} \rightarrow 0$, it is clear that the favorable pressure gradients lead to a significant relaxation of the influence of a given non-dimensional step height on $R e_{x, t}$, i.e., a larger $k / \delta_{k}^{*}$ is required for a dramatic change in the streamwise location of transition for the P8 case than either P0 or P2.

\section{Conclusions and future work}

Results from an early task in the MEATLOAF program demonstrate a strong effect of pressure gradient on the variation of transition location with respect to non-dimensional step height, $k / \delta_{k}^{*}$ that can be linked to the stability characteristics of the undisturbed flow. The excrescence design led to changes in mean velocity profile associated with blending the excrescence to zero height over the length of the insert, with earlier transition occurring for aft-facing steps. Whether the nature of the change to the boundary layer caused by the excrescence is pressure-gradient-dependent has not been addressed directly. The pressure gradients under study here lead to complicated relationships between stability and excrescence-induced transition location.

Ongoing work is investigating the effect on the flow of disturbances leading to transition in boundary layers under similar pressure gradients but with higher unit Reynolds numbers and true-step excrescences. It is hoped that the influence of disturbance geometry, displacement thickness at the step location and pressure-gradient-related stability will be further illuminated by the use of hot-wire anemometry and further quantification of the state of the perturbed boundary layer.

\section{Acknowledgments}

The authors would like to thank Lt. Jeff Komives and Dr. Carl Tilmann of the U.S. Air Force Research Laboratory for inspiring and motivating this work and Professor Mike Gaster at Queen Mary College, University of London, for the use of his Rapid- $N$ code. Contributions to the data analysis by Kevin Chen and Christine Siew are also acknowledged.

\section{References}

${ }^{1}$ van Ingen, J. L., "A suggested semi-empirical method for the calculation of the boundary layer transition region," Tech. Rep. VTH-74, Dept. of Aeron. Eng., Delft Institute of Tech., Delft, The Netherlands, 1956.

${ }^{2}$ Smith, A. M. O. and Gamberoni, N., "Transition, pressure gradient and stability theory," Tech. Rep. ES-26388, Douglas Aircraft Co., El Segundo, CA, 1956.

${ }^{3}$ Mack, L. M., "Boundary-layer stability theory," Report 709, AGARD, 1984.

${ }^{4}$ Gaster, M., "The influence of roughness on boundary layer transition," Symposium on Advances in Fluid Mechanics, Bangalore, India, July 2003, pp. 176-187.

${ }^{5}$ Klebanoff, P. and Tidstrom, K., "Mechanism by which a two-dimensional roughness element induces boundary layer transition," Phys. Fluids, Vol. 15, No. 7, 1972, pp. 1173-1188.

${ }^{6}$ Nayfeh, A., "Influence of two-dimensional imperfections on laminar flow," Paper No. 921990, SAE, 1992.

${ }^{7}$ Tani, I., "Boundary-layer transition," Annu. Rev. Fluid Mech., Vol. 1, 1969, pp. 169-196.

${ }^{8}$ Crouch, J. D., Kosorygin, V. S., and Ng, L. L., "Modeling the effects of steps on boundary-layer transition," IUTAM Symposium on Laminar-Turbulent Transition, edited by R. Govindarajan, Vol. 78 of Fluid Mechanics and its Applications, Springer Netherlands, 2006, pp. 37-44.

${ }^{9}$ Wang, Y. X. and Gaster, M., "Effect of surface steps on boundary layer transition," Expts. in Fluids, Vol. 39, 2005, pp. 679-686.

${ }^{10}$ Dryden, H. L., "Review of published data on the effect of roughness on transition from laminar to turbulent flow," $J$. Aero. Sci., Vol. 20, 1953, pp. 477-482.

${ }^{11}$ Perraud, J. and Seraudie, A., "Effects of steps and gaps on 2D and 3D transition," ECCOMAS, 2000.

${ }^{12}$ Gaster, M. and Jiang, F., "A rapid scheme for estimating transition on wings by linear stability theory," Proc. 19th Congress of ICAS, Vol. 2, Anaheim, CA, September 1994, pp. 1104-1113.

${ }^{13}$ Tani, I., "Effect of two-dimensional and isolated roughness on laminar flow," Boundary Layer and Flow Control, edited by G. Lachmann, Vol. 2, Pergamon Press, Oxford, 1961, pp. 637-656.

${ }^{14}$ Drake, A., Bender, A. M., Solomon Jr., W. D., and Vavra, A. J., "Prediction of manufacturing tolerances for laminar flow," Report AFRL-VA-WP-TR-2005-3060, Air Force Research Laboratory, 2005.

${ }^{15}$ Gaster, M., "Rapid Estimator of Eigenvalues for N-Factor Calculation," Tech. rep., Queen Mary and Westfield College, London, 1997.

${ }^{16}$ Saric, W. S. and Nayfeh, A. H., "Nonparallel stability of boundary-layer flows," Phys. Fluids, Vol. 18, No. 8, 1975, pp. $945-950$

${ }^{17}$ Schubauer, G. B. and Skramstad, H., "Laminar boundary layer oscillations and transition on a flat plate," J. Res. Nat. Bur. Standards, Vol. 38, 1947, pp. 251-292. 


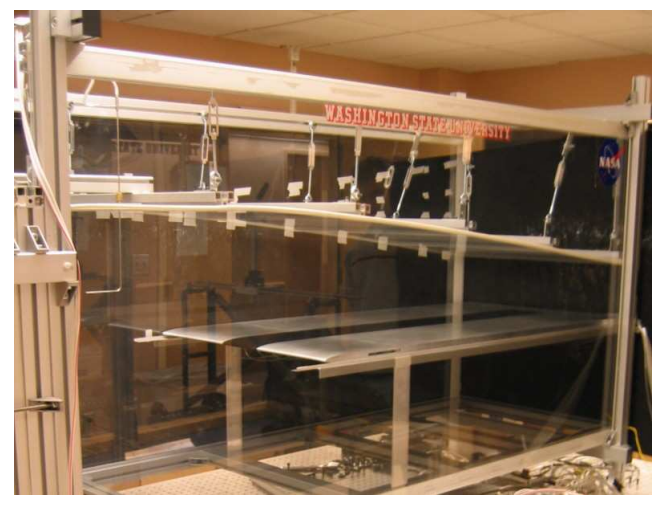

Figure 1. Flat plate in the Washington State University Low Speed Wind Tunnel.

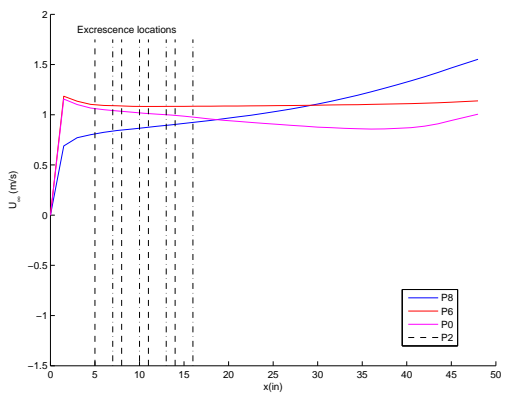

Figure 3. Streamwise distributions of pressure coefficient and freestream velocity for the three test cases. -- : forward-facing and -.-: aft-facing step locations.

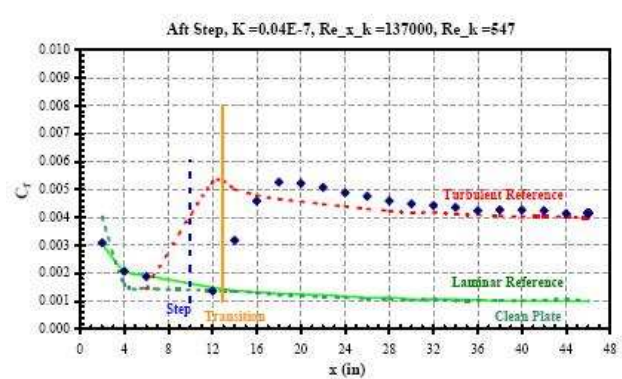

Figure 5. Sample determination of transition location from streamwise skin friction distribution.
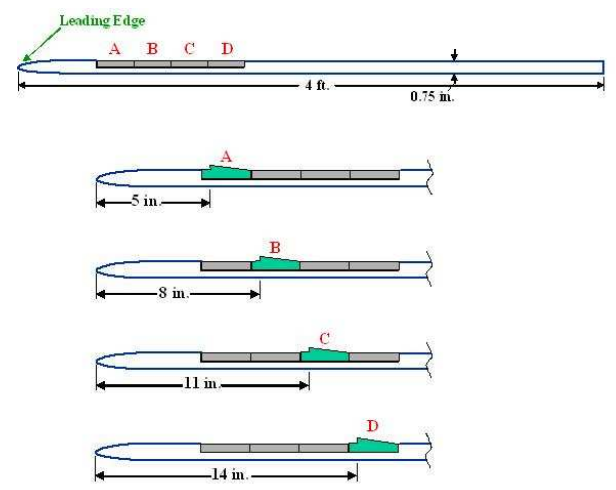

Figure 2. Schematic of the test plate and location of the excrescence inserts.

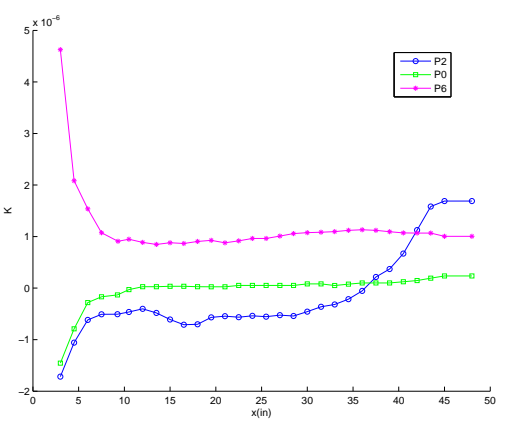

Figure 4. Distribution of acceleration parameter, $K=$ $\frac{\nu}{U_{\infty}^{2}} \frac{d U_{\infty}}{d x}$.

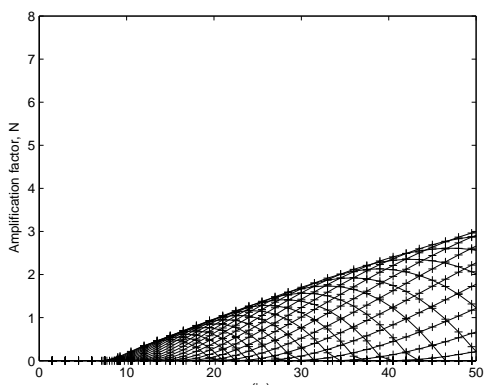

Figure 6. Rapid- $N$ calculation of amplification factor, $N$, for a Blasius boundary layer. 


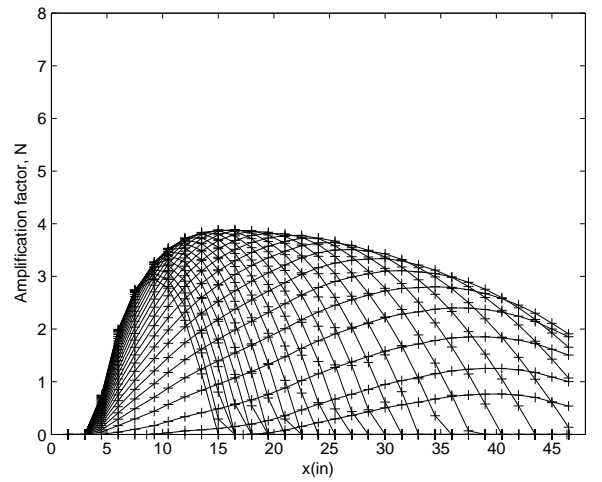

Figure 7. Rapid- $N$ calculation of amplification factor, $N$, for the P0 mildly accelerating boundary layer.

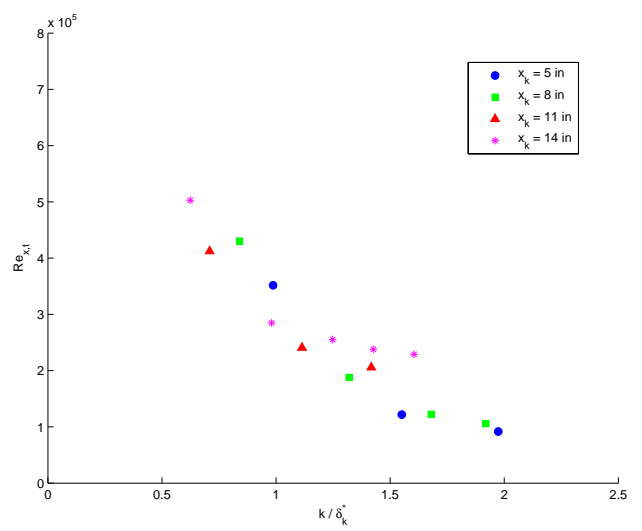

Figure 9. Variation of the transition Reynolds number, $R e_{x, t}$ with non-dimensional excrescence height $k / \delta_{k}^{*}$. Forward-facing step, P0 pressure gradient, $550 \leq$ $R e_{\delta^{*}, k} \leq 850$.

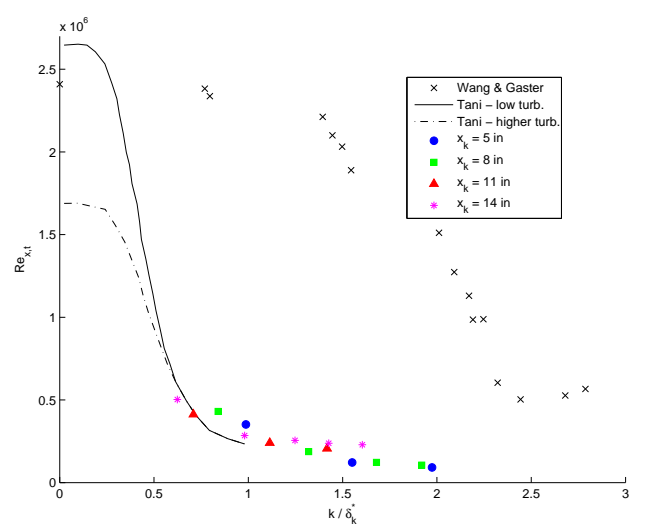

Figure 11. Comparison of the current forward-facing step results with the data of Wang \& Gaster ${ }^{9}$ for steps and $\mathrm{Tani}^{7}$ for cylindrical wires in a zero pressure gradient boundary layer.

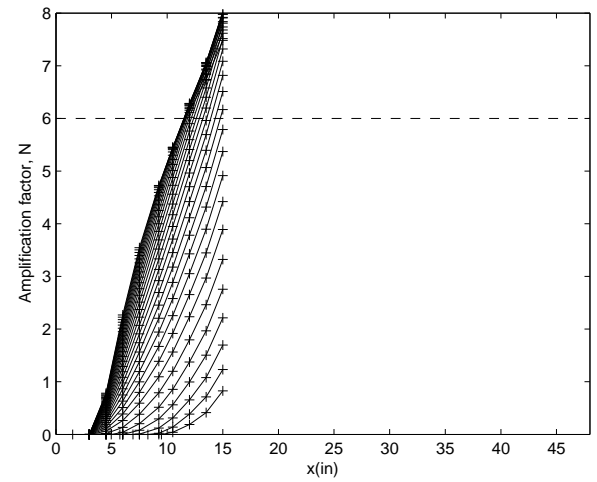

Figure 8. Rapid- $N$ calculation of amplification factor, $N$, for the P2 decelerating boundary layer.

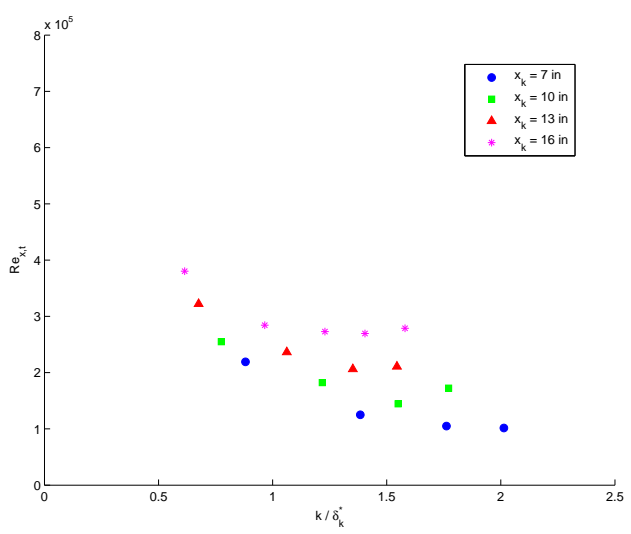

Figure 10. Variation of the transition Reynolds number, $R e_{x, t}$ with non-dimensional excrescence height $k / \delta_{k}^{*}$. Aft-facing step, P0 pressure gradient, $610 \leq$ $R e_{\delta^{*}, k} \leq 870$.

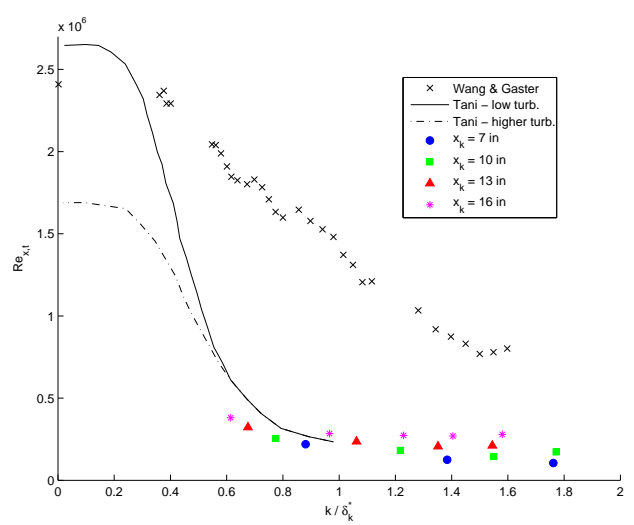

Figure 12. Comparison of the current aft-facing step results with the data of Wang \& Gaster ${ }^{9}$ for steps and Tani $^{7}$ for cylindrical wires in a zero pressure gradient boundary layer. 


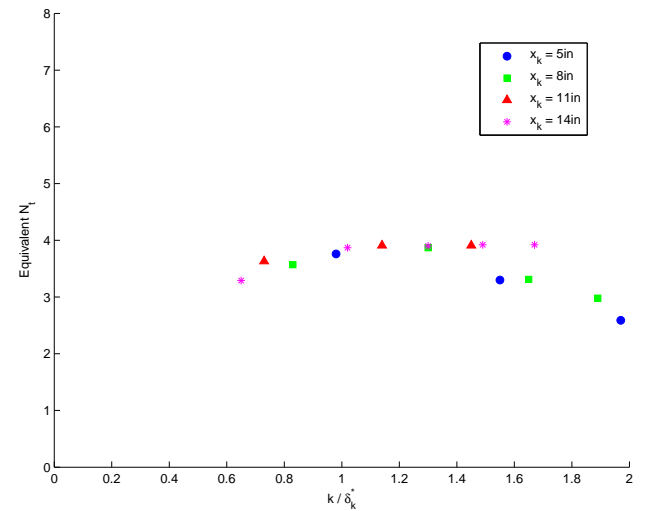

Figure 13. Variation of the transition Reynolds number, $R e_{x, t}$ with non-dimensional excrescence height $k / \delta_{k}^{*}$. Forward-facing step, P0 pressure gradient.

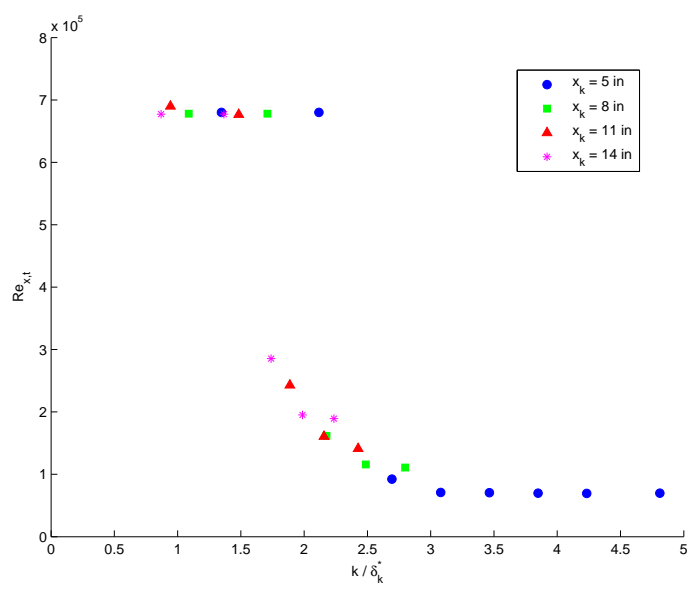

Figure 15. Variation of the transition Reynolds number, $R e_{x, t}$ with non-dimensional excrescence height $k / \delta_{k}^{*}$. Forward-facing step, P8 pressure gradient.

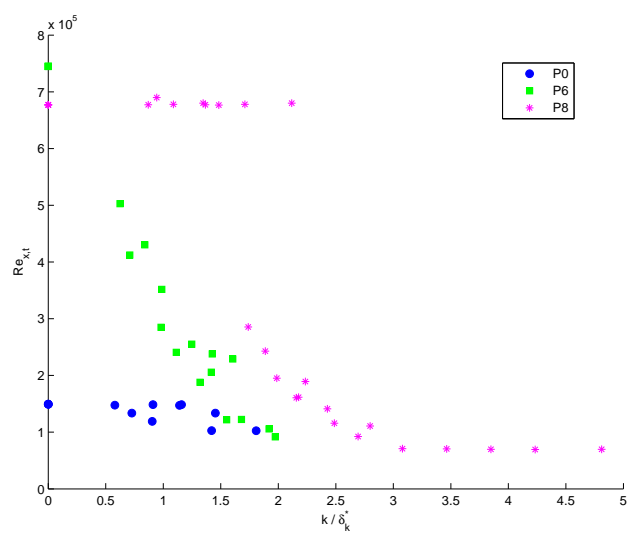

Figure 17. Comparison of forward-facing step results for all pressure gradient cases.

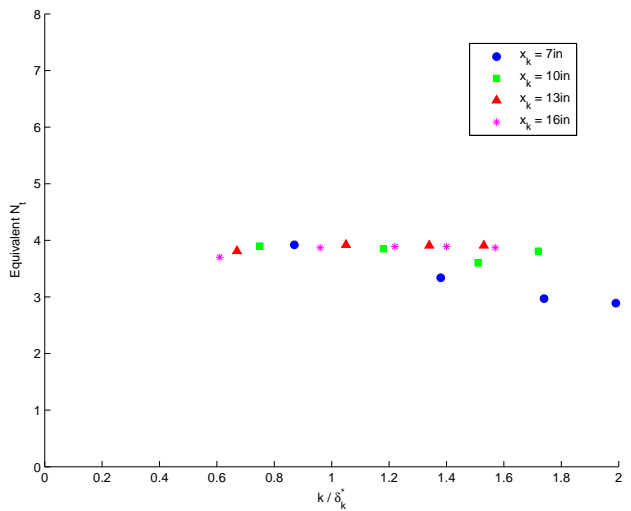

Figure 14. Variation of the transition Reynolds number, $R e_{x, t}$ with non-dimensional excrescence height $k / \delta_{k}^{*}$. Aft-facing step, P0 pressure gradient.

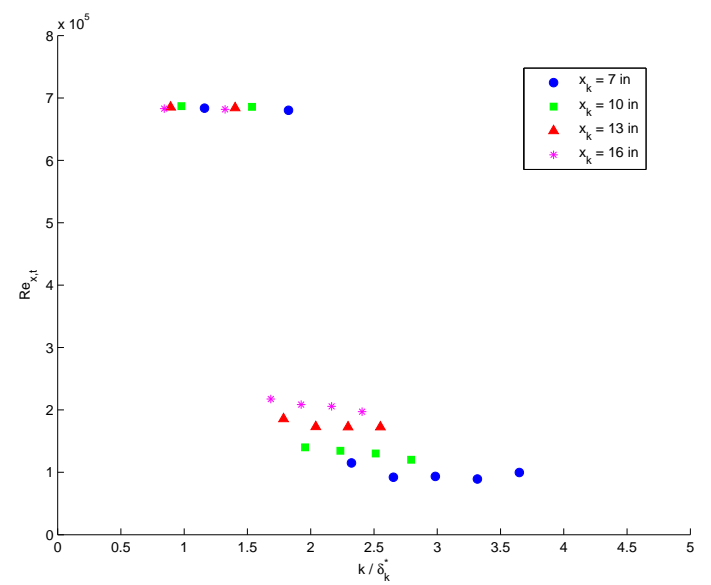

Figure 16. Variation of the transition Reynolds number, $R e_{x, t}$ with non-dimensional excrescence height $k / \delta_{k}^{*}$. Aft-facing step, P8 pressure gradient.

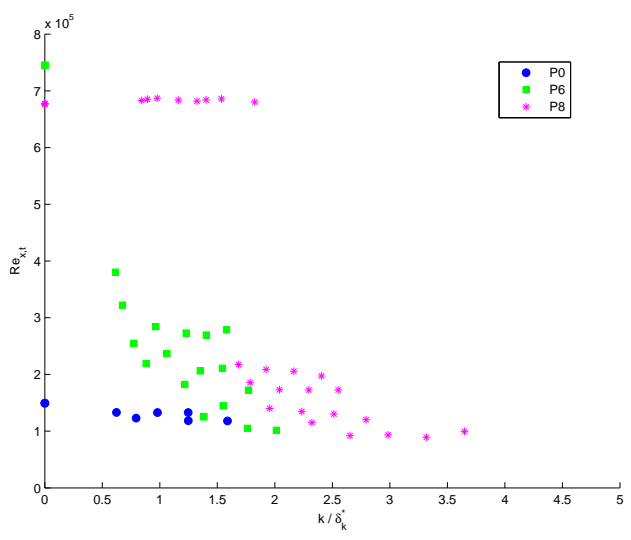

Figure 18. Comparison of aft-facing step results for all pressure gradient cases. 